\title{
Asymptomatic Rectal Perforation After Radiation for Rectal Cancer: A Diagnostic Dilemma and Role of MRI
}

\author{
Praveen Kammar, MS, MCh'; Suman Kumar Ankati, MD²; Reena Engineer, MD; \\ Prachi Patil, DM"; Vikas Ostwal, DM; Avanish Saklani, MS, FRCS ${ }^{\text {* }}$
}

Colorectal Division of Gastrointestinal Oncology, Department of Surgical Oncology, Tata Memorial Centre, Mumbai, India

Department of Radiology, Tata Memorial Centre, Mumbai, India

3 Department of Radiation Oncology, Tata Memorial Centre, Mumbai, India

${ }^{4}$ Department of Medical Gastroenterology, Tata memorial Centre, Mumbai, India

Department of Medical Oncology, Tata Memorial Centre, Mumbai, India

\begin{abstract}
Radiation-induced rectal perforation during the treatment of locally advanced rectal cancer (LARC) is very rare. Symptomatic perforation is diagnosed clinically and requires urgent intervention. However, asymptomatic perforation may be found incidentally on imaging and this can be easily confused with residual/progressive disease. Magnetic resonance imaging (MRI) may help in correct diagnosis and choosing the right line of treatment. Case 1: A 32-year-old gentleman was operated in another centre for upper rectal adenocarcinoma (T3, N0) without adjuvant therapy. He was treated with chemoradiation therapy for anastomotic site recurrence after nine months. He was referred to our centre for residual presacral disease on computerized tomography. However, MRI of the pelvis showed the mass to be heterogeneous with air pockets on T2 sequence and a fistulous communication between rectal lumen and mass. This indicated complete resolution of recurrence with a perforation at the previous colorectal anastomotic site. Case 2: A 33-year-old lady with mid rectal adenocarcinoma was treated with short course of radiotherapy and systemic chemotherapy at our centre. On reassessment using MRI, local progression was suspected. But, on careful review of MRI, a tumour site perforation was found, which was communicating with mesorectal fat. She underwent surgery for primary disease and this finding was confirmed intra-operatively. In conclusion, differentiating rectal perforation from recurrence or progressive disease during radiation for LARC is difficult due to lack of specific imaging characteristics. However, the presence of breach in the rectal wall and fistulous communication between mass and rectal lumen on MRI are confirmatory findings of perforation and thus help in choosing the right treatment.
\end{abstract}

\section{Introduction}

Radiation forms a vital part of neoadjuvant treatment in locally advanced rectal cancer (LARC) and recurrent rectal cancers. The adverse effects of radiation are well recognized; however, radiation-induced perforation at the tumour site is very rare and is poorly understood. A symptomatic rectal perforation requires an emergency surgical intervention. However, it may present silently and can give rise to suspicion of disease progression and/or residual disease on imaging. Here, we present two cases of silent perforations. Both gave rise to a considerable diagnostic dilemma, which was resolved by careful evaluation with magnetic resonance imaging (MRI).

\section{Case Reports}

\section{Case 1}

A 32-year-old gentleman was evaluated in another centre for bleeding per rectum. There were no symptoms of obstruction or distant spread and no significant family history. Clinical examination did not reveal anything significant. Colonoscopy revealed a rectal growth at $10-15 \mathrm{~cm}$ from anal verge. Biopsy was performed, and the result showed moderately differentiated adenocarcinoma. Computerized tomography (CT) scan of the abdomen and thorax showed a localized rectal growth without perirectal fat stranding or nodes and no distant metastasis. Serum carcinoembryonic antigen (CEA) was $8.3 \mathrm{ng} / \mathrm{ml}$. He underwent upfront open anterior resection with covering ileostomy. Postoperative recovery was uneventful. Histopathology revealed moderately differentiated adenocarcinoma with extracellular mucin pools at places. Circumferential resection margins (CRM) and proximal and distal resection margins were free. Pathological staging was T3, N0 (out of 22 nodes). Adverse histological features were absent. He did not receive any adjuvant treatment. Nine months after the surgery, he developed pain abdomen. Clinical examination did not yield anything significant. Serum CEA was $7 \mathrm{ng} / \mathrm{ml}$. CT scan showed a $7 \times 4.8 \times 5.6 \mathrm{~cm}$ lesion posterior to the rectum at the anastomotic site

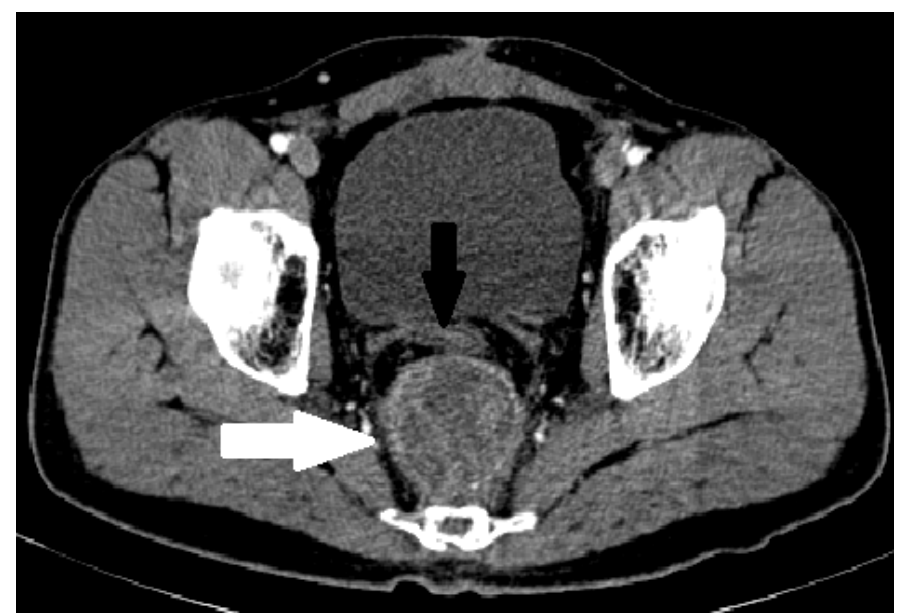

Figure 1. Contrast-enhanced CT scan image at the level of the bladder, done after CRT for local recurrence, showing a $5 \times 5 \mathrm{~cm}$ well-defined heterogeneously enhancing (white arrow) mass posterior to the rectal tube (black arrow). CRT, chemoradiation; CT, computerized tomography.

with preaortic nodes of $2.3 \times 1.6 \mathrm{~cm}$ size, with no other metastases. He received local radiation ( $50 \mathrm{~Gy}$ ) with capecitabine followed by 2 cycles of capecitabine and oxaliplatin (CAPOX). Post-treatment showed that he was clinically asymptomatic with a falling CEA trend (7 to $4.8 \mathrm{ng} / \mathrm{ml}$ ). A repeat CT scan done 3 months later showed persistent $5 \times 5 \mathrm{~cm}$, well-defined, enhancing, hyperdense mass in the presacral region abutting the posterior wall of rectum (Figures 1 and 2). He was then referred to our cancer centre. No remarkable abnormalities were observed in the digital rectal examination. 

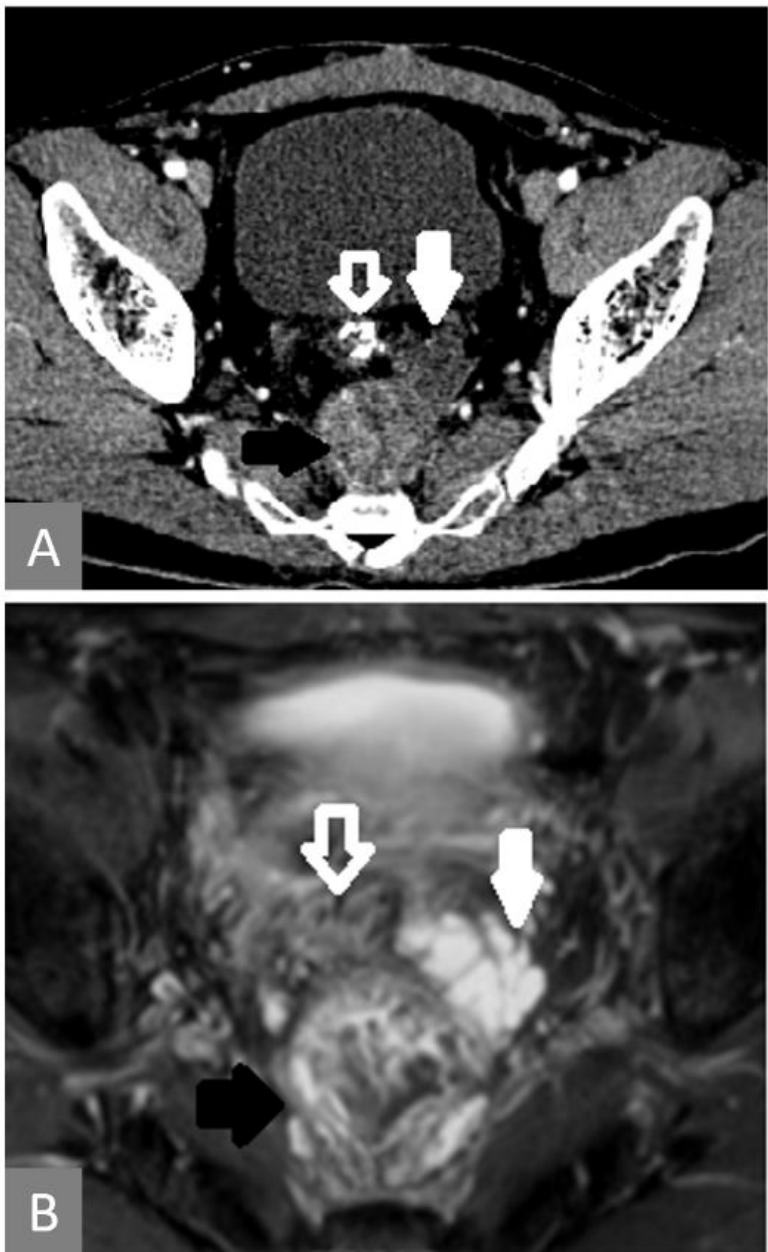

Figure 2. T2-MRI showing that the enhancing lesion seen on $\mathrm{CT}$ is heterogeneous on MRI (solid black arrow) and the nonenhancing lesion in CT is mucin deposit, which is T2 intense (solid white arrow). Rectal tube indicated with white line arrow in both images. (A) CT showing the enhancing mass in the presacral region (solid black arrow) along with another nonenhancing mass anterolateral to it (solid white arrow). (B) MRI of the lesion at the same level. CRT, chemoradiation; CT, computerized tomography; MRI, magnetic resonance imaging.

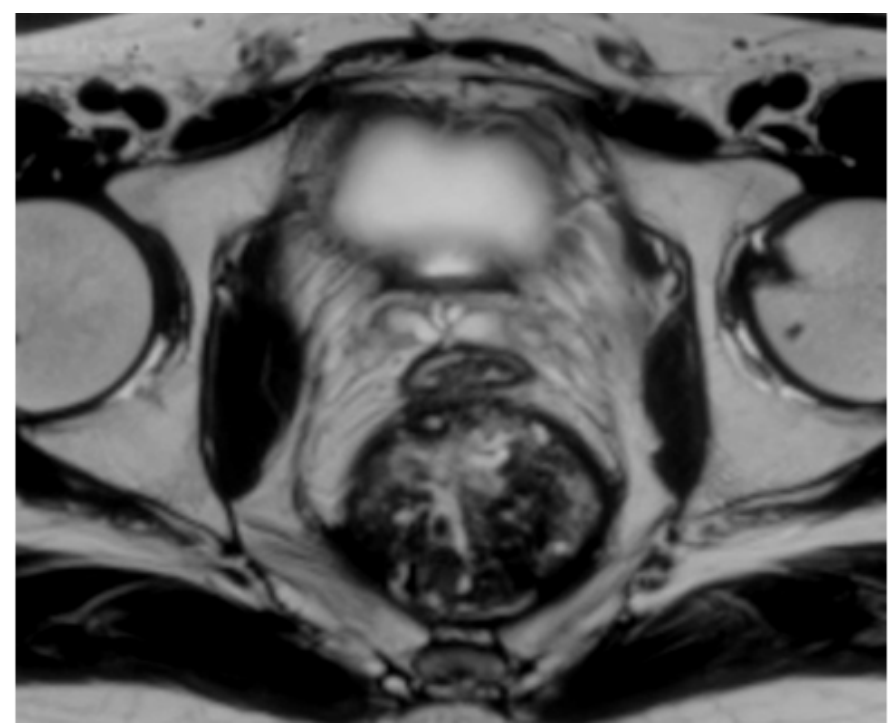

Figure 3. Breach in the rectal wall and communication between the presacral mass and the rectal tube.
CT-guided biopsy of the mass was inconclusive. An MRI was done to rule out residual disease (Figures 2,3) and it showed a heterogeneously T2-intense presacral mass communicating with the rectal lumen, suggestive of breakdown at the site of anastomosis (Figure 3). It was concluded that the recurrent tumour at the anastomotic site had responded completely and eventually led to a subclinical anastomotic leak. The patient was advised close follow-up. However, four months later, the patient developed a diffuse peritoneal disease, which was not amenable to cytoreductive surgery. He was started on palliative chemotherapy with FOLFIRI (folinic acid, fluorouracil, and irinotecan) regimen. At 6 months follow-up, the patient was alive with disease.

\section{Case 2}

A 33-year-old premenopausal female presented with complaints of constipation and bleeding per rectum for 4 months. There were no symptoms of distant spread. There was no history of rectal or gynaecological cancers in the family. Digital examination showed growth at $5 \mathrm{~cm}$ from the anal verge. Endoscopic biopsy confirmed mucinous adenocarcinoma. CT scan of abdomen and thorax showed a suspicious para-aortic node and a suspicious liver metastasis. CEA was $11.4 \mathrm{ng} / \mathrm{ml}$. MRI of pelvis showed a transmural tumour in the mid-rectum with the involvement of the CRM, but the vaginal wall appeared free. She underwent short-course radiation ( 25 Gy, 5 fractions, over 5 days) followed by 4 cycles of chemotherapy with 5-fluorouracil, leucovorin, irinotecan, and oxaliplatin (FOLFIRINOX). Post-radiation MRI done 5 weeks later showed a new $5.5 \times 6.8 \times 5.8 \mathrm{~cm}$ heterogeneous exophytic mass arising from the left posterolateral wall of the rectum, displacing it (Figure 4). On T2 sequence, there were bright areas inside the lesion, suggestive of mucin collection. Careful evaluation of MRI images revealed a breach in the rectal wall communicating rectal lumen with the mass. Heterogeneity of the mass was due to necrotic debris and air pockets secondary to perforation. The mesorectal fascia was involved in the anterior planes. The liver lesion turned out to be a simple cyst and the para-aortic node showed complete resolution.

The patient underwent anterior resection with hysterectomy for R0 resection along with diversion transverse colostomy. Intraoperatively, it was observed that there was a contained perforation at the tumour site posteriorly without peritoneal contamination. Postoperatively, the patient developed anastomotic leak necessitating a Hartmann's procedure. Histopathology showed no residual tumour. However, 2 out of 16 nodes showed mucinous adenocarcinoma deposits with perinodal extension. There were pools of extracellular mucin in mesorectal fat. She received adjuvant chemotherapy of 6 cycles CAPOX. When she came back for stoma closure, she had suspicious deposits on the anterior peritoneum. On exploration, there was extensive peritoneal disease not amenable to surgical treatment. Hence, stoma reversal was not done. She was started on palliative chemotherapy. At 28 months follow-up, the patient is alive with disease.

\section{Discussion}

Acute adverse effects of neoadjuvant radiation in LARC include gastrointestinal toxicity, genitourinary, neurological complications, and delayed wound healing. However, most of these are self-limiting [1]. Severe bleeding, strictures, perforation, fistula, and bowel obstruction occur in the chronic phase, which may not become apparent for months to years [2]. Rectal perforation and anastomotic breakdowns are very rare. Only a handful of case reports are seen in the medical literature (Table 1).

All the reported cases of rectal cancer perforation have been T3 or T4 tumours with transmural involvement. It seems to be a combination of tumour characteristics and radiation effects that lead to perforation since most of the transmural tumours do not perforate. A larger tumour has a hypoxic and hypovascular centre leading to central necrosis [3,4], while radiation accelerates cell lysis in well-perfused peripheral zone, thus leading to weakening of the wall. However, there is anecdotal evidence that larger and undifferentiated tumours may be less responsive to radiation $[5,6]$.

Mucinous variety of adenocarcinomas, as seen in both the cases presented here, are known to be less responsive to neoadjuvant radiation and have poor outcome overall. Mucin is produced and stored under 

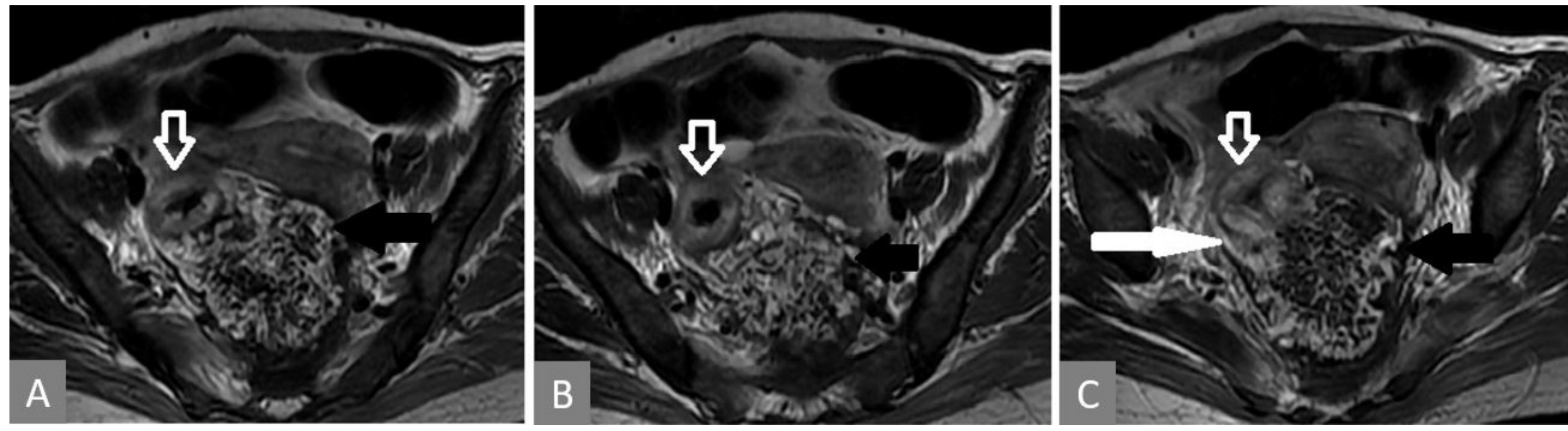

Figure 4. Serial T2 weighted MRI images (in cranio-caudal direction). Thickened rectal wall (white hollow arrow) with heterogeneous exophytic mass (solid black arrow) pushing it (A, B, and C). Posterior breach is observed in the rectal wall (white solid arrow in panel C).

\begin{tabular}{|c|c|c|c|c|c|c|c|}
\hline \multicolumn{2}{|l|}{ Case Report } & Timing of Radiation & Type of Radiation & Presentation & Location of Tumor & Locally Advanced Stage & Surgical Intervention \\
\hline \multirow[t]{4}{*}{ Lee et al. [7] } & Case 1 & 5 days later & CRT & Symptomatic & Transmural (BPR) & Yes & Conservative \\
\hline & Case 2 & 1 month later & CRT & Symptomatic & Transmural (BPR) & Yes & Ileostomy \\
\hline & Case 3 & 2 weeks later & CRT & Symptomatic & Trnasmural (APR) & Yes & Colectomy \\
\hline & Case 4 & 4 weeks later & CRT & Symptomatic & Transmural (APR) & Yes & Colectomy \& lleostomy \\
\hline \multicolumn{2}{|c|}{ Takase et al. [11] } & $\begin{array}{l}\text { In the middle of } \\
\text { radiation }\end{array}$ & CRT & Symptomatic & Transmural (BPR) & Yes & Sigmoid Colostomy \\
\hline \multicolumn{2}{|c|}{ Khan et al. [12] } & 1 week later & CRT & Symptomatic & Transmural (APR) & Yes & No \\
\hline \multicolumn{2}{|c|}{ El Gendy et al. [13] } & 2 weeks later & CRT & Symptomatic & Not at Tumor Site (BPR) & Yes & Low Anterior Resection \\
\hline
\end{tabular}

APR, above peritoneal reflection; BPR, below peritoneal reflection; CRT, chemoradiation.

pressure, which leads to mucin perforating rectal wall and migrating into the surrounding space wherever the wall weakens.

The time period of presentation has been varied (Table 1). All the reported cases were symptomatic, thus requiring some form of intervention. In Case 1, there was a diversion stoma, and in Case 2, the perforation might have been small, hence both the cases were clinically silent. Complication of rectal perforation could be dangerous. Out of four patients in the report of Lee et al., two died in the preoperative period [7].

Short course radiotherapy (SCRT) is said to be safer than long course radiotherapy [8,9], but rectal perforation after SCRT is also reported [10]. The potential risks for CRT-related rectal cancer perforation may include the presence of diverticula, collagenases, and tumour ulceration [11].

Small bowel obstruction, fistula, cystitis, and chronic diarrhoea are some of the late side effects of re-irradiation [12-18]. Fistulae in the lower pelvis are often associated with recurrent disease and may be exacerbated by tumour destruction [17]. Here, in Case 1, radiotherapy (RT) was used only after pelvic recurrence. The presacral recurrence showed excellent response to RT, but later images showed a leak from the rectum, most probably from the anastomotic line. This might be due to RT-induced ischemic changes of the normal rectal tissue.

In asymptomatic cases, CT and MRI are essential tools to differentiate recurrence from perforation and other benign findings, as fine-needle aspiration or biopsy are not always helpful. However, they still have limitations. The post-rectal surgery CT picture can be varied, wherein a presacral mass could be anything from normal postoperative changes to hematoma, abscess, uterus or enlarged prostate, and local recurrence. It is vital to remember that the normal postoperative changes that occur in the midline become well-defined over a period of time [19]. Similar findings were seen in Case 1. The size of the mass and its attenuation value could not be used to conclusively diagnose the recurrence $[20,21]$.

MRI is more sensitive than CT in detecting recurrences but not without caveats. As seen in both the cases here, a recurrent tumour typically shows a T2 hyperintense signal but so do hematoma, granulation tissue, and inflammation [22]. A T2 hypointense lesion creates further confusion.
In both the cases, the diagnosis of perforation was possible due to the demonstration of communication between the mass and rectal lumen on MRI, the absence of mucin in the mass while it was present in the surrounding sites along with normal CEA, and lack of symptoms. Prophylactic diversion stoma for tumours above peritoneal reflection is suggested by some [12], but doing it for all cases for such a rare complication is not justified.

\section{Conclusion}

Perforation of rectal tumour is a rare event during the treatment of rectal cancer. It may present asymptomatically and after a considerable time lapse, incidentally found on imaging. No specific imaging characters are defined to differentiate rectal perforation from recurrence or progressive disease, however, identifying a breach in the rectal wall and fistulous communication between mass and rectal lumen on MRI are confirmatory findings of perforation and thus help in choosing the right treatment. There are two major learning points in the report: (1) rectal tumour perforation during radiation of rectal cancer is rare but can occur and create diagnostic dilemma, especially in clinically silent cases; (2) a well-timed MRI helps in differentiating perforation from a residual/progressive disease.

\section{Article Information}

*Correspondence: Avanish Saklani, MS, FRCS

Colorectal Division of Gastrointestinal Oncology, Department of Surgical Oncology, Tata Memorial Centre, Dr. E Borges Road, Parel, Mumbai 400 012, India. Email: asaklani@hotmail.com

Received: Sep. 15, 2017; Accepted: Nov. 20, 2017; Published:Jan. 26, 2019

DOI: $10.24983 /$ scitemed.acri.2019.00095

Copyright (c) 2019 The Author(s). This is an open-access article distributed under the terms of the Creative Commons Attribution 4.0 International License (CC-BY). 


\section{Funding: None}

Conflict of Interest: None

\section{Keywords}

Cancer; complication; magnetic resonance imaging; radiation; rectum.

\section{References}

1. Glimelius B, Grönberg H, Järhult J, Wallgren A, Cavallin-Ståhl E. A systematic overview of radiation therapy effects in rectal cancer. Acta Oncol Stockh Swed 2003;42(5-6):476-492.

2. Do NL, Nagle D, Poylin VY. Radiation proctitis: current strategies in management. Gastroenterol Res Pract 2011;2011:917941.

3. Baek JH, Jang JE, Kang CM, Chung HY, Kim ND, Kim KW. Hypoxia-induced VEGF enhances tumor survivability via suppression of serum deprivation-induced apoptosis. Oncogene 2000;19(40):4621-4631.

4. Ferrara N. Vascular endothelial growth factor: basic science and clinical progress. Endocr Rev 2004;25(4):581-611.

5. Lin AY, Wong WD, Shia J, et al. Predictive clinicopathologic factors for limited response of T3 rectal cancer to combined modality therapy. Int J Colorectal Dis 2008;23(3):243-249.

6. Das P, Skibber JM, Rodriguez-Bigas MA, et al. Predictors of tumor response and downstaging in patients who receive preoperative chemoradiation for rectal cancer. Cancer 2007;109(9):1750-1755.

7. Lee J, Chen F, Steel M, Keck J, Mackay J. Perforated rectal cancer associated with neoadjuvant radiotherapy: report of four cases. Dis Colon Rectum 2006;49(10):1629-1632.

8. Sauer $\mathrm{R}$, Becker $\mathrm{H}$, Hohenberger $\mathrm{W}$, et al. Preoperative versus postoperative chemoradiotherapy for rectal cancer. $N$ Engl J Med 2004;351(17):1731-1740.

9. Bujko K, Nowacki MP, Nasierowska-Guttmejer A, Michalski W, Bebenek $\mathrm{M}, \mathrm{Kryj} \mathrm{M}$. Long-term results of a randomized trial comparing preoperative short-course radiotherapy with preoperative conventionally fractionated chemoradiation for rectal cancer. Br J Surg 2006;93(10):1215-1223.

10. Martel G, Al-Suhaibani Y, Stern H, Boushey RP. Rectal cancer perfo- ration after short-course neoadjuvant radiotherapy. Dis Colon Rectum 2007;50(10):1724-1725; author reply 1726.

11. Takase N, Yamashita K, Sumi Y, et al. Local advanced rectal cancer perforation in the midst of preoperative chemoradiotherapy: a case report and literature review. World J Clin Cases 2017;5(1):18-23.

12. Khan A, Al-Suhaibani Y, Al-Sharief A. Rectal cancer perforation: a rare complication of neo-adjuvant radiotherapy for rectal cancer. Int J Oncol 2009;7(2):1-5.

13. ElGendy K. Rectal perforation after neoadjuvant chemoradiotherapy for low-lying rectal cancer. BMJ Case Rep 2015;2015.

14. Lingareddy V, Ahmad NR, Mohiuddin M. Palliative reirradiation for recurrent rectal cancer. Int J Radiat Oncol Biol Phys 1997;38(4):785790.

15. Mohiuddin M, Marks G, Marks J. Long-term results of reirradiation for patients with recurrent rectal carcinoma. Cancer 2002;95(5):11441150.

16. Ng MKY, Leong T, Heriot AG, Ngan SYK. Once-daily reirradiation for rectal cancer in patients who have received previous pelvic radiotherapy. J Med Imaging Radiat Oncol 2013;57(4):512-518.

17. Das $P$, Delclos ME, Skibber JM, et al. Hyperfractionated accelerated radiotherapy for rectal cancer in patients with prior pelvic irradiation. Int J Radiat Oncol Biol Phys 2010;77(1):60-65.

18. Koom WS, Choi Y, Shim SJ, et al. Reirradiation to the pelvis for recurrent rectal cancer. J Surg Oncol 2012;105(7):637-642.

19. Kelvin F, Korobkin M, Heaston D, Grant J, Akwari O. The pelvis after surgery for rectal carcinoma: serial CT observations with emphasis on nonneoplastic features. Am J Roentgenol 1983;141(5):959-964.

20. Reznek RH, White FE, Young JWR, Fry IK, Nicholls RJ. The appearances on computed tomography after abdomino-perineal resection for carcinoma of the rectum: a comparison between the normal appearances and those of recurrence. Br J Radiol 1983;56(664):237-240.

21. Moss AA, Thoeni RF, Schnyder P, Margulis AR. Value of computed tomography in the detection and staging of recurrent rectal carcinomas. J Comput Assist Tomogra 1981;5(6):870-874.

22. Messiou C, Chalmers AG, Boyle K, Wilson D, Sagar P. Pre-operative MR assessmentofrecurrentrectalcancer.BrJ Radiol 2008;81(966):468-473. 